\title{
Stochastic effects in a compartmental model for mitotic checkpoint regulation
}

\author{
Bashar Ibrahim ${ }^{1}$, Peter Dittrich ${ }^{1}$, Stephan Diekmann², Eberhard Schmitt ${ }^{2, a}$ \\ ${ }^{1}$ Jena Centre for Bioinformatics \& Institute of Computer Science, Friedrich-Schiller-University \\ Jena, D-07737 Jena, Germany. http: / / www . minet.uni-jena.de \\ ${ }^{2}$ Leibniz Institute for Age Research - FLI, Beutenbergstraße 11, D-07745 Jena, Germany. \\ http://www.fli-leibniz.de
}

\begin{abstract}
Summary
The proper segregation of sister chromatids at onset of anaphase is surveyed by the mitotic spindle assembly checkpoint. The concentration dynamics of the complexes APC:Cdc20 and MCC:APC determine exit from metaphase to anaphase. We have developed a model based on 14 proteins and complexes to describe concentration dynamics by ordinary differential equations in three compartments coupled by diffusion. One kinetochore in each compartment determines the attachment status to the spindle pole. Here, we focus on the role of noise in the segregation surveillance process. The deterministic differential equations are enriched by a stochastic term adding white noise of different amplitudes. Obviously, for the known physiological parameter ranges, noise does not disturb the checkpoint function. On the other hand, there is a connection between diffusion and noise, that could become important when considering a larger number of chromosomes.
\end{abstract}

\section{Introduction}

The proper segregation of sister chromatids in metaphase to anaphase transition is essential for maintaining the integrity of the genome in mitosis [17]. Accuracy in this mechanism is needed to ensure that aneuploidy is avoided, which causes birth defects, contributes to cancer [3] and is possibly a cause of tumorigenesis [16]. During evolution, the segregation process has been rather strictly conserved and many proteins involved therein have analogs or homologs in all kingdoms.

To understand the molecular basis of such a monitoring mechanism, Ibrahim et al. [5] have developed a model to simulate the dynamics of proteins and complexes which regulate the mitotic spindle checkpoint. This deterministic model describes qualitatively the checkpoint mechanism by a system of compartmental ordinary differential equations and was validated by comparison to results from deletion and overexpression experiments.

Although most cellular processes proceed in a spatially and temporally ordered fashion, not all noise is rejected [12]. Moreover, it has become clear that the role of intrinsic noise in cellular functions may be complex and cannot always be treated as a small perturbation to the deterministic behavior [14]. Different studies have explored the influence of molecular fluctuations on complex processes and cases have been found where noise seems to be essential for the

\footnotetext{
${ }^{\mathrm{a} C}$ Corresponding author: email: eschmitt@ fli-leibniz.de
} 
required biological function [13]. In addition, this type of modeling is becoming increasingly important in the field of systems biology, e.g. for modeling the cell cycle and gene regulation $[13,1,2,14,6,7]$. Cell cycle control is subject to fluctuations from different sources $[1,13]$ in a natural environment.

This study focuses on the investigation of the role of noise in the deterministic model derived in [5]. Here, we make use of stochastic differential equations (SDEs) to simulate in particular the implications of molecular fluctuations on the Mitotic Spindle Assembly Checkpoint $\left({ }^{M} S A C\right)$. Regarding the concentrations of [APC:Cdc20] and [MCC:APC] as measurements for the switch triggering exit from metaphase to anaphase, we model the dynamics of these concentrations in dependency of attachment states of the involved kinetochores by a deterministic desciption of a network of 14 proteins and protein complexes which regulate the dynamics of [APC:Cdc20] and [MCC:APC]. Only when the last kinetochore attaches, the APC:Cdc20 complex is allowed to form and thus starts a signalling cascade leading to onset of anaphase. Adding white noise to the deterministic equations allows the investigation of fluctuation effects.

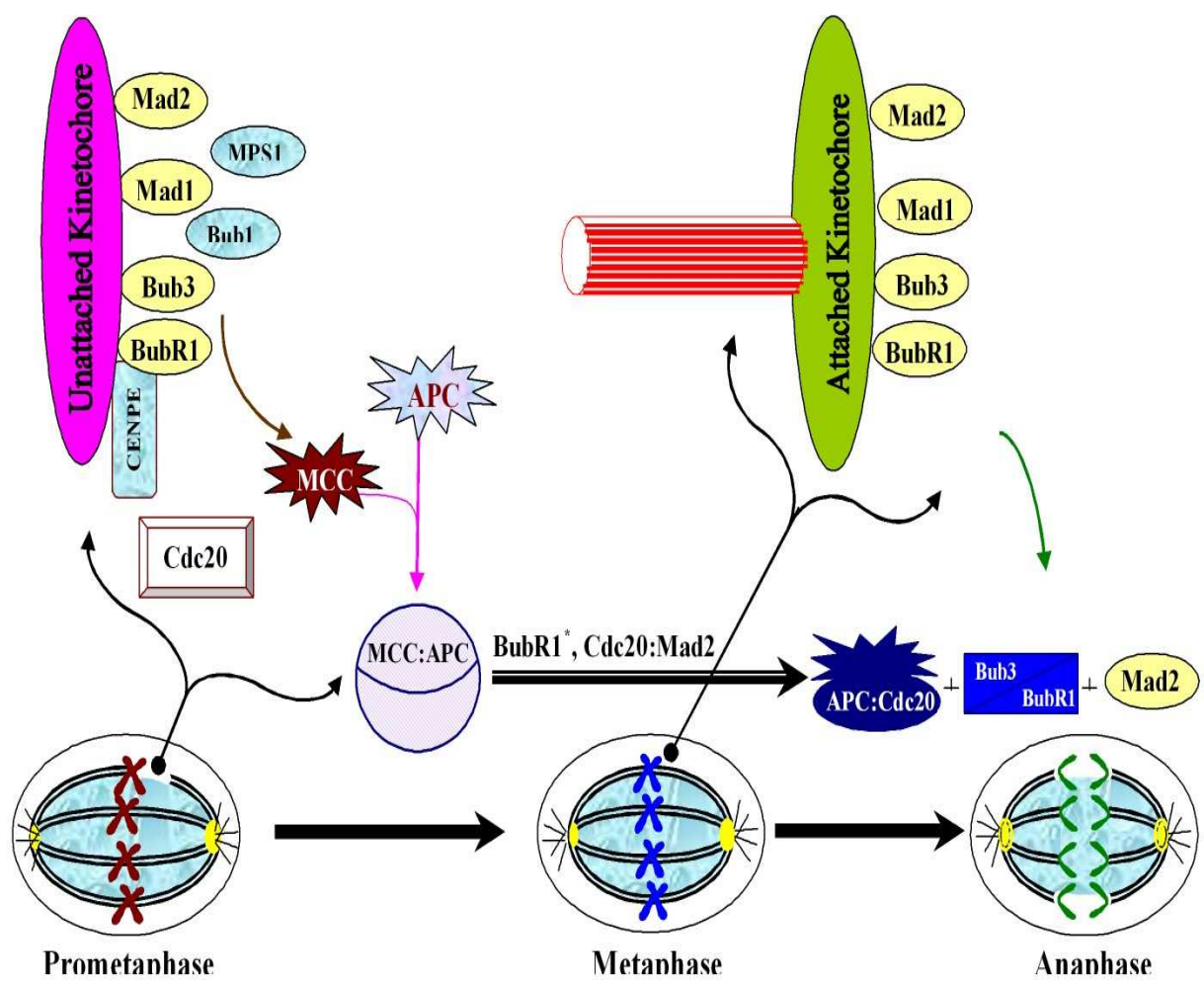

Figure 1: Scheme of reactions and localizations. As long as a kinetochore is unattached, MPS1, Bub1, CENPE, (and further players) mediate activation reactions at the kinetochore (upper left). When the kinetochore is attached, these reactions stop (upper right). As long as there is a single unattached kinetochore, MCC and APC form a complex (lower left, prometaphase to metaphase). When the last kinetochore has attached, the APC:Cdc20 complex can form and triggers exit to anaphase (lower right).

\section{The Model and its Simulation}

In our model, we consider 14 proteins and protein complexes, called species here, namely Mad2, Mad1, BubR1, Bub3, Mad2*, Mad1*, BubR1*, BubR1:Bub3, APC, Cdc20, MCC, 


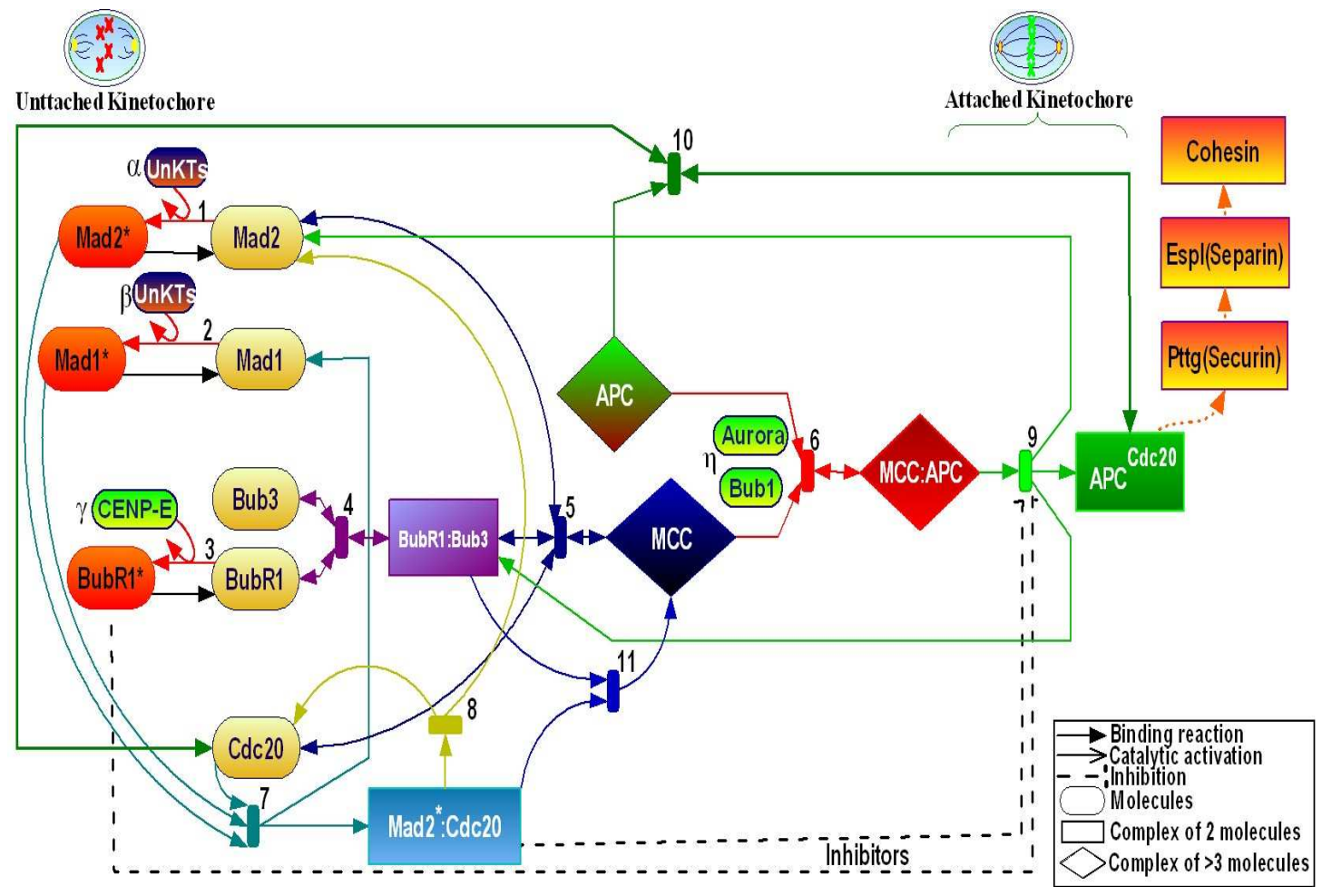

Figure 2: Reaction network for one compartment. The activation reactions with parameters $\alpha, \beta$, and $\gamma$ continue, as long as the kinetochore is unattached (left). When the respective kinetochore attaches, $\alpha, \beta$, and $\gamma$ are set to 0 . A network of reactions leads to the formation of the MCC:APC complex (middle). These reactions continue as long as there is at least one unattached kinetochore. This is the consequence of the diffusion in the system, because the respective kinetochore does not any longer supply activated Mad1*, Mad2*, and BubR1*. When the last kinetochore attaches, the APC:Cdc20 complex begins to form and leads to a cascade of reactions cutting cohesin in the end and initiating anaphase (right).

MCC:APC, Cdc20:Mad2, and APC:Cdc20. A detailed biomolecular description of the reactions involved can be found in [5]. In an illustration (Figure 1), we show the proteins, indicating also their localization in the reaction process. Here, we summarize the reaction equations. Three activation reactions are mediated by the kinetochore:

$$
\begin{gathered}
\operatorname{Mad} 2 \underset{\mathrm{k}_{-1}}{\stackrel{\alpha}{\rightleftharpoons}} \operatorname{Mad} 2^{*} \\
\operatorname{Mad} 1 \underset{\mathrm{k}_{-2}}{\stackrel{\beta}{\rightleftharpoons}} \operatorname{Mad} 1 *^{*} \\
\operatorname{BubR} 1 \underset{\mathrm{k}_{-3}}{\stackrel{\gamma}{\rightleftharpoons}} \text { BubR1* }
\end{gathered}
$$

The forward reactions with kinetic constants $\alpha, \beta$, and $\gamma$ (Table 1) in the unattached state stop when the kinetochore attaches. Therefore, in the attached state, we put $\alpha=\beta=\gamma=$ 0 . Therefore, Mad2*, Mad1*, and BubR1* are not any longer produced by activation at the respective kinetochore, but they are still supplied by diffusion from other compartments with unattached kinetochores. The backward reactions as well as all other reaction equations are not 
influenced by the attachment status of the kinetochore:

$$
\begin{aligned}
& \text { BubR1 + Bub3 } \underset{\mathrm{k}_{-4}}{\stackrel{\mathrm{k}_{4}}{\rightleftharpoons}} \text { BubR1:Bub3 } \\
& \operatorname{Mad} 2+\operatorname{BubR} 1: \operatorname{Bub} 3+\operatorname{Cdc} 20 \underset{\mathrm{k}_{-5}}{\stackrel{\mathrm{k}_{5}}{\rightleftharpoons}} \mathrm{MCC} \\
& \mathrm{MCC}+\mathrm{APC} \underset{\mathrm{k}_{-6}}{\stackrel{\mathrm{k}_{6}}{\rightleftharpoons}} \mathrm{MCC}: \mathrm{APC} \\
& \operatorname{Mad} 2 *+\operatorname{Mad} 1 *+\operatorname{Cdc} 20 \stackrel{k_{7}}{\longrightarrow} \text { Cdc20:Mad2 + Mad1 } \\
& \text { Cdc20:Mad2 } \stackrel{k_{8}}{\longrightarrow} \mathrm{Cdc} 20+\operatorname{Mad} 2 \\
& \text { MCC:APC } \underset{\substack{\text { BubR1 } \\
\text { Cdc20:Mad2 }}}{\stackrel{\text { Inhibitors }}{\longrightarrow}} \text { APC:Cdc20 + BubR1:Bub3 + Mad2 } \\
& \text { APC:Cdc20 } \underset{\mathrm{k}_{-9}}{\stackrel{\mathrm{k}_{9}}{\rightleftharpoons}} \mathrm{APC}+\mathrm{Cdc} 20 \\
& \text { Cdc20:Mad2 + BubR1:Bub3 } \stackrel{\mathrm{k}_{10}}{\longrightarrow} \text { MCC }
\end{aligned}
$$

The reaction equations define ordinary differential equations for the concentrations of the respective molecules. Mass action kinetics is assumed for reaction rules (1)-(8) and (10)-(11). Not much is known about MCC:APC dissociation (9), so we have used a mixed inhibition function of Michaelis-Menten type:

$$
f_{I n h}(S, I)=\frac{\mathrm{V} * S}{k_{m} *\left(1+\frac{I}{k_{i s}}\right)+S *\left(1+\frac{I}{k_{i c}}\right)}
$$

where $S \equiv$ [MCC:APC] is the substrate, $I \equiv\left[\mathrm{BubR} 1^{*}\right]+[\mathrm{Cdc} 20: \operatorname{Mad} 2]$ is the inhibitor concentration, $\mathrm{V}$ is the forward maximum velocity, $\mathrm{k}_{m}$ is the forward Michaelis-Menten constant, $\mathrm{k}_{i s}$ is the specific (competitive) inhibition constant, and $\mathrm{k}_{i c}$ is the catalytic (noncompetitive) inhibition constant.

Here, we give examples for two of the 14 ordinary differential equations, namely for the concentration of the complexes MCC:APC and APC:Cdc20 :

$$
\begin{aligned}
& \frac{\mathrm{d}[\mathrm{MCC}: \mathrm{APC}]}{\mathrm{dt}}=\mathrm{k}_{6}[\mathrm{MCC}][\mathrm{APC}]-\mathrm{k}_{-6}[\mathrm{MCC}: \mathrm{APC}]-f_{\text {Inh }}(S, I) \\
& \frac{\mathrm{d}[\mathrm{APC}: \mathrm{Cdc} 20]}{\mathrm{dt}}=f_{I n h}(S, I)-\mathrm{k}_{10}[\mathrm{APC}: \mathrm{Cdc} 20]+\mathrm{k}_{-10}[\mathrm{APC}][\mathrm{Cdc} 20]
\end{aligned}
$$

The equations described so far show the evolution in time of the concentrations of the 14 species near one kinetochore. To concentrate on the main effects, we consider three compartments $\mathrm{X}$, $\mathrm{Y}$, and $\mathrm{Z}$ in a linear spatial arrangement, so that molecules can only diffuse between $X$ and $Y$, and between $Y$ and $Z$. Each compartment contains one kinetochore, which can be attached or not attached to the spindle. As mentioned before, the attachment of the spindle within a specific compartment is represented as a change of parameters $\alpha, \beta$, and $\gamma$ in that compartment according to the values in Table 1 (unattached) and the value 0 (attached). 
Instead of introducing spatial variables, we use Fick's first law to describe diffusion between compartments. In our simulation with discretized time, diffusion is then discribed by a characteristic constant $\Omega=\frac{D}{l^{2}}[11,15]$ that multiplies the concentration differences. We obtain for a species $i$ and reaction terms $R_{i, A}$ in compartment A the following equations:

$$
\begin{aligned}
d[i]_{X} / d t & =\Omega\left([i]_{Y}-[i]_{X}\right)+R_{i, X} \\
d[i]_{Y} / d t & =\Omega\left([i]_{X}-2[i]_{Y}+[i]_{Z}\right)+R_{i, Y} \\
d[i]_{Z} / d t & =\Omega\left([i]_{Y}-[i]_{Z}\right)+R_{i, Z}
\end{aligned}
$$

Because many biological details are yet unknown, we set $\Omega=1 \mathrm{~s}^{-1}$, assuming a diffusion constant of $D=0.01 \mu \mathrm{m}^{2} \mathrm{~s}^{-1}$ and a distance of $l=10 \mu \mathrm{m}$ between compartments for all substances. This estimate is based on the known diffusion constants.

In these equations, subscripts $\mathrm{X}, \mathrm{Y}$, and $\mathrm{Z}$ denote the concentrations of the specific species in the respective compartment. Hence, the complete system consists of 42 concentration functions of time as solution of a system of 42 ordinary differential equations. Note, that attachment states of the three compartments are additional parameters. Beginning with initial concentrations taken from literature (c.f. [5]), the equations are integrated with all attachment parameters in the state "unattached" (symbolized by UUU) until equilibrium is reached. Then, attachment parameter of compartment $X$ is turned on (UUA), and the three activation reactions (1)-(3) in $X$ are set to zero. After equilibrium is reached again, attachment in compartment $Y$ is turned on (UAA) until equlibrium is reached. Finally, all three kinetochores are attached (AAA). These symbols are also used in Figures 3-5, where they designate the end of the simulation time of the respective phase.

For integration, those kinetic constants, which are documented in the literature, are used directly, whereas for all other constants physiologically reasonable values are substituted. This is done for many choices, and performance of the model is optimized according to an objective function measuring the concentration of APC:Cdc20, which should be 0 for the first three phases UUU, UUA, UAA, and should rise to a maximal value in phase AAA within a time scale of about 3 to 5 minutes. The optimization details can be found in [5]. As a result, the values in Table 1 were found as optimal values for the kinetic parameters. They are also used in our perturbation model here.

\begin{tabular}{|ll|lll||lll|ll|}
\hline$\alpha$ & $=4.82$ & $\mathrm{k}_{-1}=0.00752$ & $\mathrm{k}_{7}=10.0$ & & \\
\hline$\beta=9.92$ & $\mathrm{k}_{-2}=0.00100$ & $\mathrm{k}_{8}=0.00235$ & & \\
\hline$\gamma=6.08$ & $\mathrm{k}_{-3}=9.83$ & $\mathrm{k}_{9}=0.00271$ & $\mathrm{k}_{-9}=10.0$ \\
\hline $\mathrm{k}_{4}=0.412$ & $\mathrm{k}_{-4}=3.44$ & $\mathrm{k}_{10}=10.0$ & & \\
\hline $\mathrm{k}_{5}=0.00100$ & $\mathrm{k}_{-5}=0.00100$ & $\mathrm{~V}=0.0197$ & $\mathrm{k}_{i c}=6.84$ \\
\hline $\mathrm{k}_{6}=10.0$ & $\mathrm{k}_{-6}=0.00100$ & $\mathrm{k}_{m}=9.21$ & $\mathrm{k}_{i s}=0.00100$ \\
\hline
\end{tabular}

Table 1: Kinetic parameters used for simulation

As we do not have any special information about the origin of fluctuations in the cell cycle $[13,1]$, we assume random extrinsic fluctuations, mathematically described by white noise $[12,4,1]$. For each species $i$ in compartment $j$, the stochastic Langevin-type equation has the 
following form:

$$
\frac{d[i]_{j}}{d t}=C(i)_{j}+W_{i}(t) \sqrt{2 \cdot N_{i} \cdot[i]_{j}}
$$

where $C(i)_{j}$ is the original right-hand side of the deterministic equation and $W_{i}(t)$ is the (multiplicative) Gaussian white noise for species $i$ with zero mean and unit variance

$$
\left\langle W_{i}(t)\right\rangle=0, \quad\left\langle W_{i}(t) W_{i}\left(t^{\prime}\right)\right\rangle=\delta\left(t-t^{\prime}\right) .
$$

$N=N_{i}$ denotes the noise amplitude parameter, which, for simplicity, is the same for all species.

We computed the concentration functions for several values of $N$. Here, we show the results for $N=10^{-8}$ (almost no noise) and $N=10^{-5}$. We used $\Omega=1$ as in the deterministic simulation, but in addition, to simulate the influence of diffusion on the system, we used $\Omega=0.01$ and $\Omega=0.005$.

The simulations were performed using standard numerical techniques for stochastic differential equations (including the strong implicit 1.5 order Runge-Kutta method [8], and the explicit Runge-Kutta method of order 1.5 [8]). We implemented our code in MATLAB [10].

\section{Results}

In Figure 3, the concentration curves for APC:Cdc20 and MCC:APC complexes are shown for a time period of about 12 minutes. The noise amplitude is $N=10^{-8}$. In all simulations, steady state conditions (regarding noise, this means constant average of concentration) are reached within less than 3 minutes in all 3 phases (UUU, UUA, UAA) with at least one unattached kinetochore. For $\Omega=1$ (top), there are no visible differences between the concentration curves for compartments $\mathrm{X}, \mathrm{Y}$, and Z (data not shown). Diffusion is obviously fast enough to level out the small differences induced by noise. In addition, after steady state is reached in phase UUU, the concentrations remain at that level during phases UUA and UAA. The graphs are almost identical to the concentration curves in the deterministic simulation without noise ( [5]). For $\Omega=0.01$ (middle), first differences appear in phases UUA and UAA as compared to UUU: the equilibrium value is rising. Also in phase AAA, the onset of APC:Cdc20 increase and MCC:APC decrease is much faster. Also, concentration dynamics differences between compartments X, Y, and $\mathrm{Z}$ become visible (data not shown). For $\Omega=0.005$ (bottom), these effects become even more pronounced.

In Figure 4, showing the same concentration functions in the same ordering of $\Omega$, the noise level is much higher at $N=10^{-5}$. Whereas for $\Omega=1$ the overall shapes of the curves still resemble the curves for low noise level, there is a large overshoot of [MCC:APC] $(\Omega=0.01)$ in phase UUU, and, for $\Omega=0.005$, [MCC:APC] decays very rapidly already in phase UAA. It is also questionable, whether the concentration reached is enough to prevent exit from metaphase to anaphase. These effects increase for even higher $N$-values and disrupt mitotic control. To give an impression of the different behaviour in the three compartments, we have included Figure 5 which shows the respective dynamics in these compartments for $N=10^{-5}$ and $\Omega=0.005$. 
[APC:Cdc20] in Y-compartment
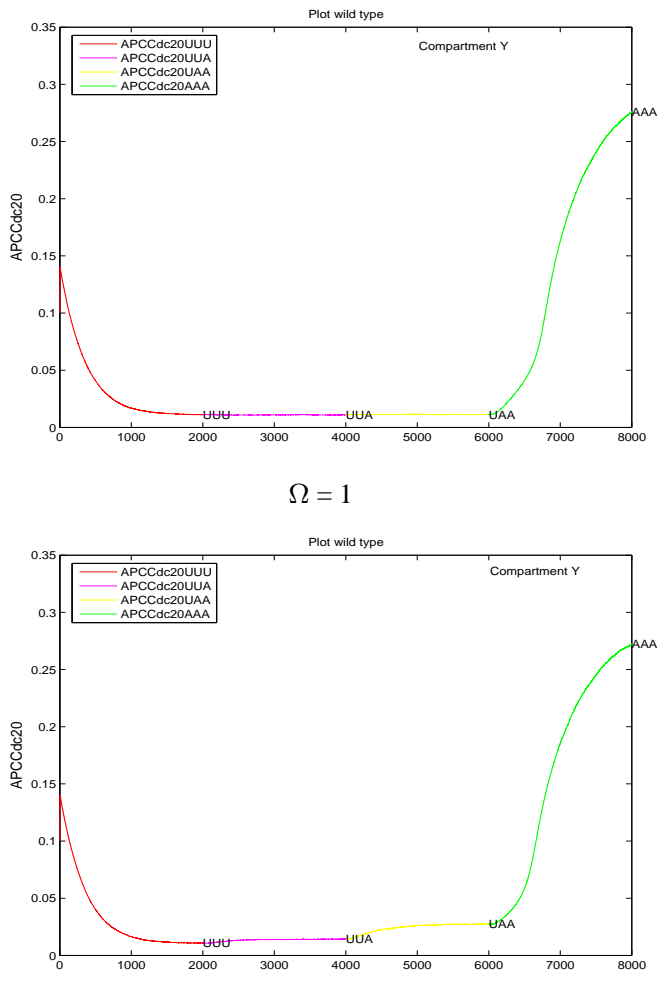

$\Omega=0.01$

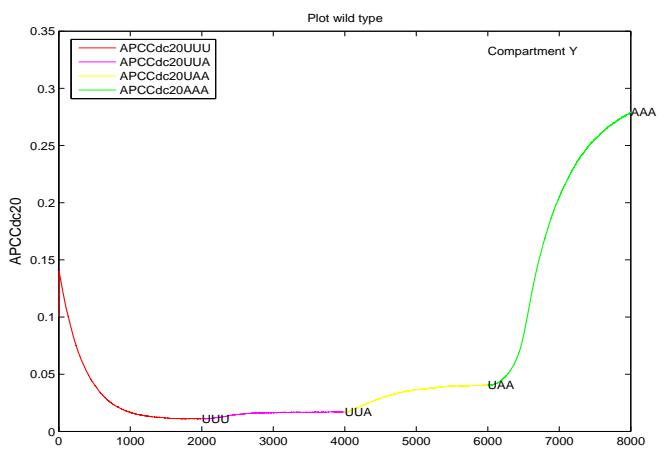

$\Omega=0.005$
[MCC:APC] in Y-compartment

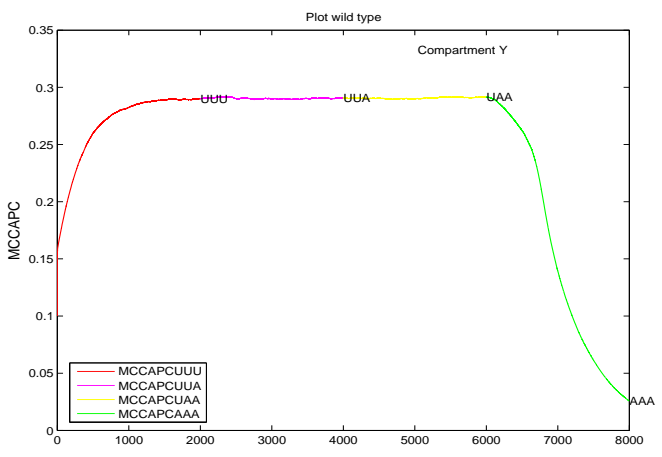

$\Omega=1$

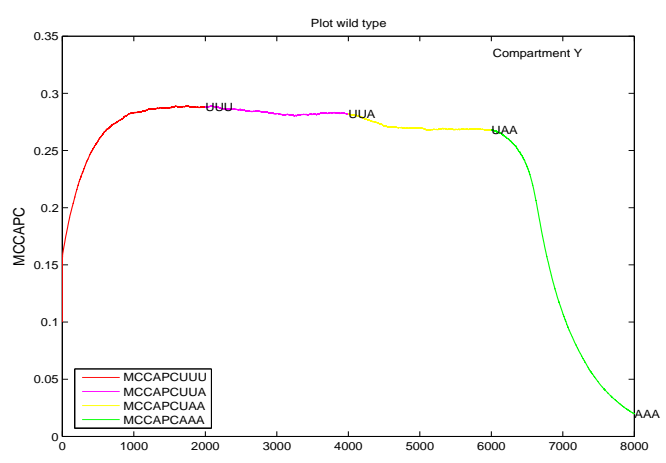

$\Omega=0.01$

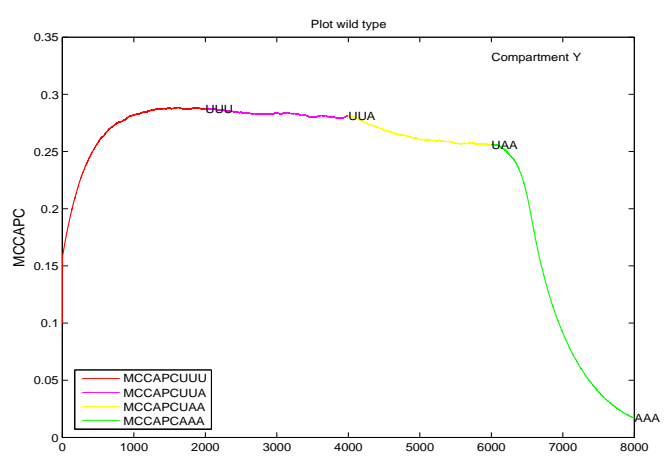

$\Omega=0.005$

Figure 3: Concentration of APC:Cdc20 (left) and MCC:APC (right) as a function of time (8000 iterations corresponding to $12 \mathrm{~min}$ ) in the middle compartment $\mathrm{Y}$ at noise amplitude $N=10^{-8}$. The first part (orange, from time 0 to 'UUU') of the curves describes the phase with three kinetochores Unattached (UUU); the second part (red, 'UUU'-'UUA'), the phase with two kinetochores Unattached and one Attached (UUA); etc. (cf. insert). With decreasing diffusion coefficient $\Omega$ (cf. text), the concentration differences within the 3 phases UUU, UUA, UAA, increase more and more. Steady state is reached after at most $3 \mathrm{~min}$. When all kinetochores are attached (green curve 'UAA'-'AAA', phase AAA), APC:Cdc20 rises immediately and MCC:APC decays.

\section{Discussion and Conclusion}

The proper segregation of sister chromatids at onset of anaphase is surveyed by the mitotic spindle assembly checkpoint. The concentration dynamics of the complexes APC:Cdc20 and MCC:APC determine exit from metaphase to anaphase. Our model, describing concentration dynamics by ordinary differential equations, is based on 14 proteins and complexes, which de- 
[APC:Cdc20] in Y-compartment
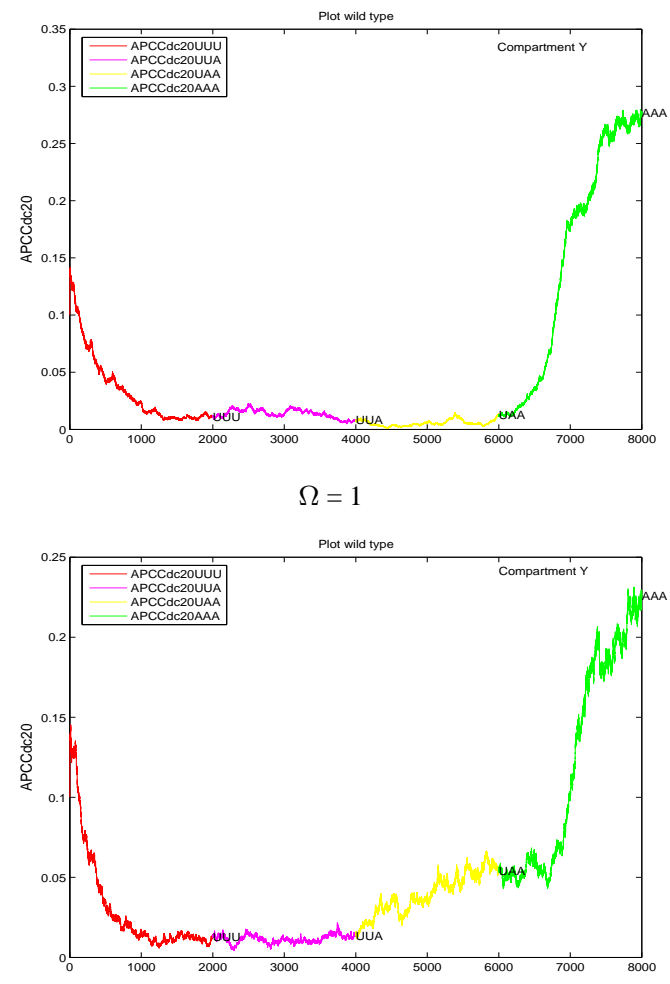

$\Omega=0.01$

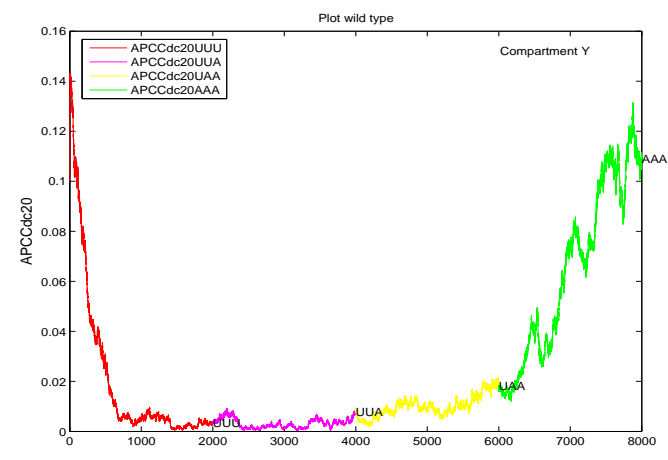

$\Omega=0.005$
[MCC:APC] in Y-compartment

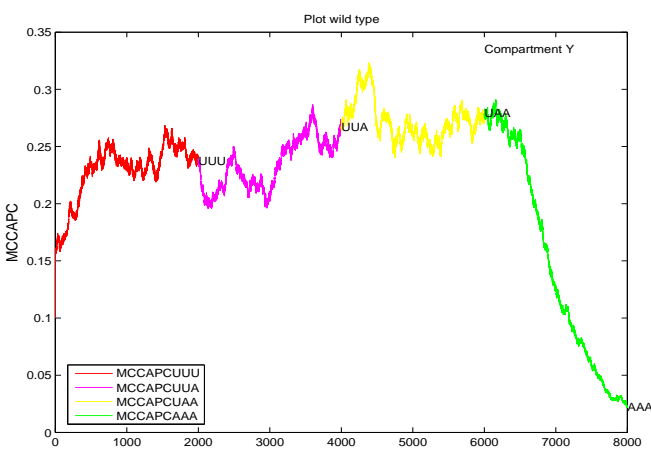

$\Omega=1$

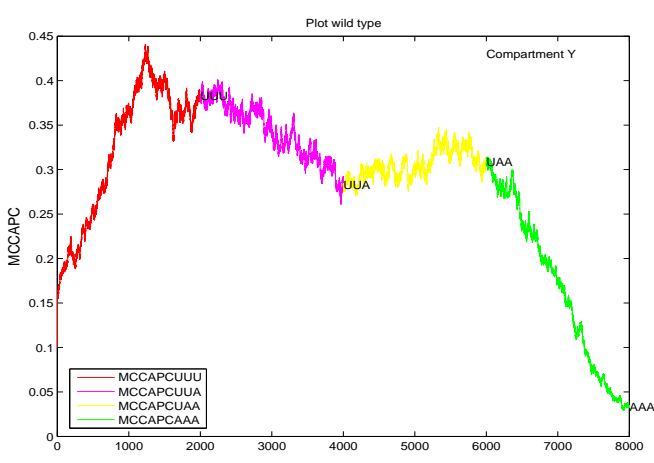

$\Omega=0.01$

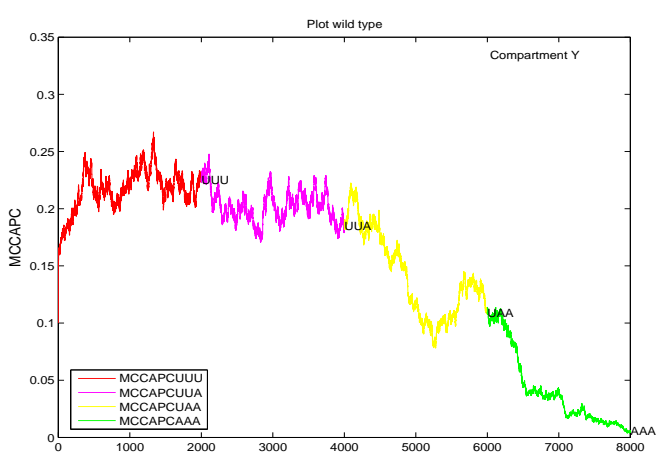

$\Omega=0.005$

Figure 4: Concentration of APC:Cdc20 (left) and MCC:APC (right) as a function of time (8000 iterations corresponding to $12 \mathrm{~min}$ ) in the middle compartment $\mathrm{Y}$ at noise amplitude $N=10^{-5}$. With decreasing diffusion coefficient $\Omega$, dynamics in the phases with one kinetochore unattached (UAA) become more and more distinct, and in some cases a steady state equilibrium is not reached any more within phase simulation time $(3 \mathrm{~min})$.

termine increase or decrease of the two key complexes near a kinetochore, dependent on its attachment status. To investigate the interaction of concentrations near different kinetochores, we have coupled three compartments by diffusion. As for many reaction constants only ranges, but no exact values, are known, we optimized a particular objective function based on the expected dynamics of APC:Cdc20 concentration in [5], where the details of this procedure can be found. The result was a set of concentration parameters and initial values, allowing the simulation of the deterministic model showing a correct behaviour of the spindle checkpoint and also reproducing overexpression and deletion effects [5]. The model is based on mass action laws for all reactions except one (9), which is described by a mixed Michaelis-Menten kinetic. This 
may be an oversimplification, because non-linear effects may be involved. On the other hand, this argument applies to almost all reactions considered here. It is even questionable, how diffusion can be described correctly, as ongoing research shows that certain signalling molecules seem to travel along one dimensional fibres. So, the formulation of the reaction equations might be only a first step. However, for example the mixed Michaelis-Menten kinetic, we used here, has several parameters which were optimized and could in this way provide a description which is close to the actual reaction.

As in natural processes noise is a never neglectable ingredient, we have addressed here the question, whether noise has a destructive influence, or, as in [13], is even essential for the correct function. We therefore take the same description by differential concentration equations for the single compartments and also the same geometric arrangement of three compartments as in [5]. But here, the deterministic differential equations are enriched by a stochastical term adding white noise of different amplitudes.

We could show that the spindle checkpoint dynamics as described by our model is stable with respect to extrinsic white noise to a rather large extend. Only for a noise amplitude larger than $N=10^{-4}$, the function of the checkpoint is disrupted. The influence of noise, on the other hand, is dependent on the diffusion coefficient. Whereas diffusion in the known physiological parameter ranges is fast enough to level out concentration differences in the distinct compartments, noise is able to build up considerably different dynamics near different kinetochores when the diffusion constant is decreased. This effect could become important when considering more chromosomes, which is necessary to simulate human checkpoint behaviour.

We did not find indications that noise is essential for the checkpoint function, in contrast to the effects of noise in the cell cycle as a whole, where noise seems to prevent dynamics from getting stuck in singular points [13]. Our results suggest that a fast diffusion, as in the physiologically relevant parameter ranges, helps to stabilize the system and to prevent noise to disrupt the checkpoint function. Along these lines it will be interesting to investigate the system using methods developed in [9]. They show, using a master equation approach, that in certain metabolic networks noise is not propagated, but has only local influence. These findings coincide with ours on a global level. On the other hand, we did not yet investigate the influence of a single perturbation on the system, e.g. by a concentration impulse response, as they suggest. But this will be a challenging task for us for the future.

In the model presented here, a linear arrangement of three compartments was used. This is only a first approximation to a more complex model for 46 chromosomes, arranged in a two dimensional mitotic equatorial plate, where each inner kinetochore has six neighbours - for mathematical symmetry. The outside kinetochores in this geometry have also connection to an outer region without any kinetochores, which is also connected to all kinetochores via the third dimension. Presently, we are modeling this situation, which is much more involved. There are still debates about the exact ranges of diffusion coefficients, and also the dimensionality is in question. There are hints for one dimensional diffusion in certain cases, possibly enabled by diffusion along fibres. In further investigations, we will therefore use different diffusion constants for distinct reactants and also try to simulate different dimensionalities. A further goal is also the investigation of the influence of the geometry (e.g. in the mitotic equatorial plate) on the checkpoint dynamics using partial differential equations. Here, the localization of the reactions and reactants to the kinetochore, on the one hand side, or to the 'nucleosol', on the other hand side, may play an important role. 
[APC:Cdc20] in compartments X,Y,Z

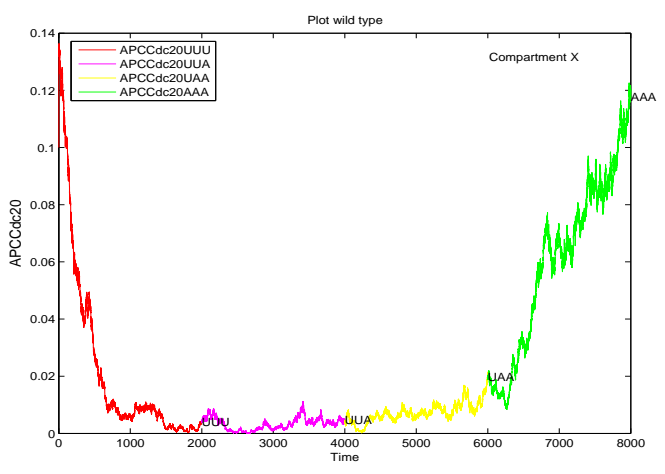

$\mathrm{X}$

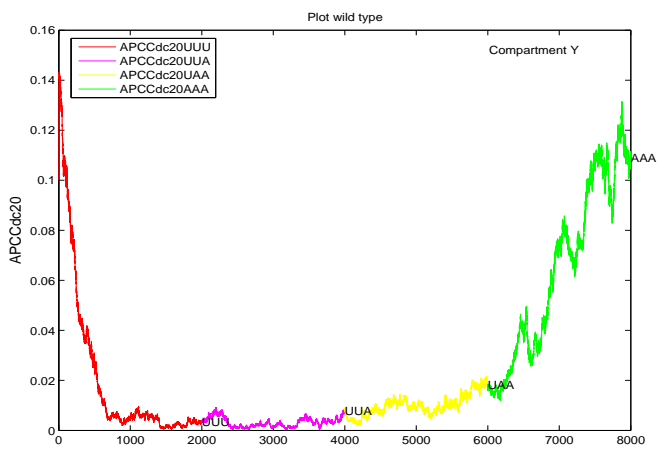

Y

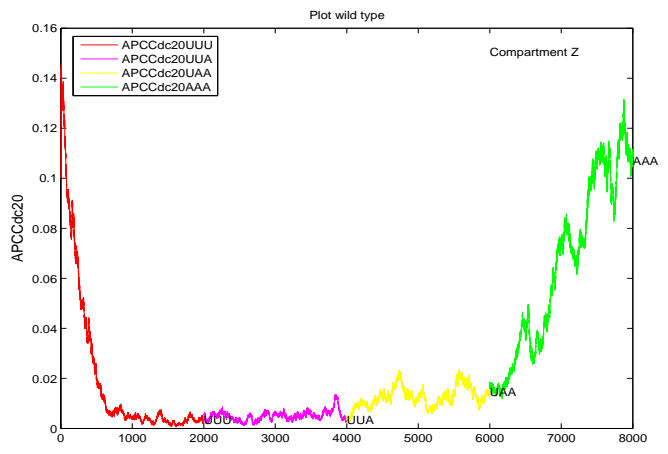

$\mathrm{Z}$
[MCC:APC] in compartments X,Y,Z

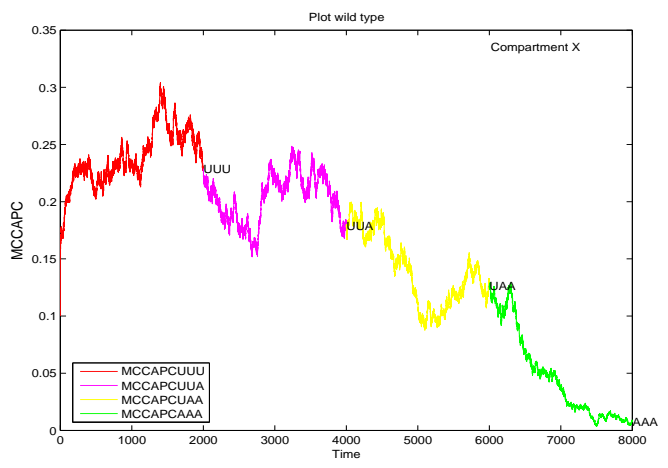

$\mathrm{X}$

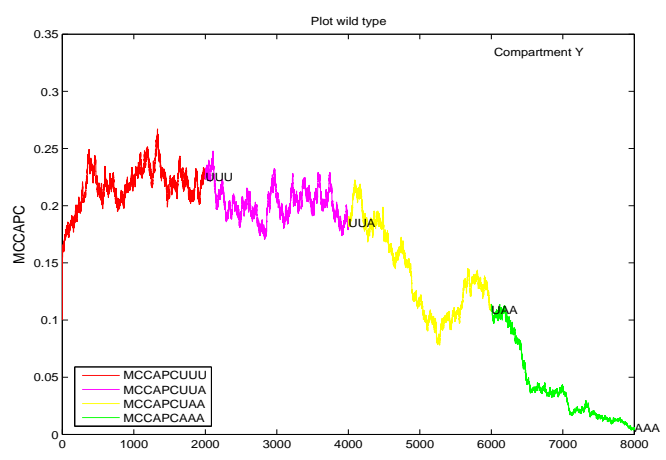

Y

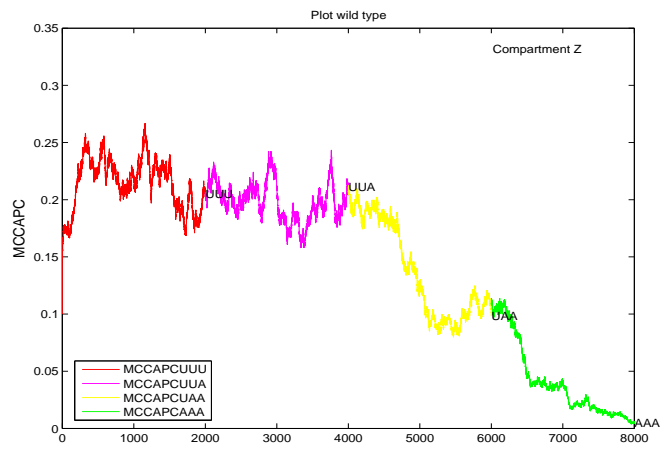

Figure 5: Concentration of APC:Cdc20 (left) and MCC:APC (right) in the three compartments $\mathbf{X}, \mathbf{Y}, \mathbf{Z}$, at a noise amplitude of $N=10^{-5}$ for diffusion coefficient $\Omega=0.005$. For these parameters, strong differences in the [APC:Cdc20] and [MCC:APC] dynamics in the different compartments become visible.

\section{Acknowledgements}

Bashar Ibrahim was supported by the German Academic Exchange Service (DAAD, grant A/04/31166). We would also like to thank the unknown reviewers for helpful comments. 


\section{References}

[1] D. Battogtokha and J. J. Tyson. Bifurcation analysis of a model of the budding yeast cell cycle. CHAOS, 14(3):653-661, September 2004.

[2] K.-C. Chen, T.-Y. Wang, H.-H. Tseng, C.-Y. F. Huang, and C.-Y. Kao. A stochastic differential equation model for quantifying transcriptional regulatory network in saccharomyces cerevisiae. Bioinformatics, 21(12):2883-2890, 2005.

[3] D. A. Compton. Chromosomes walk the line. Nat Cell Biol, 8(4):308-10, 2006.

[4] D. T. Gillespie. The chemical Langevin and Fokker-Planck equations for the reversible isomerization reaction. J. Phys. Chem. A, 106:5063-5071, 2002.

[5] B. Ibrahim, S. Diekmann, E. Schmitt, and P. Dittrich. Qualitative in-silico model of the mitotic spindle assembly checkpoint. submitted, pages 1-27, 2007.

[6] M. Kaern, T. C. Elston, W. J. Blake, and J. J. Collins. Stochasticity in gene expression: From theories to phenotypes. Nature, 6:451-464, June 2005.

[7] T. B. Kepler and T. C. Elston. Stochasticity in transcriptional regulation: Origins, consequences, and mathematical representations. Biophysical Journal, 81:3116-3136, December 2001.

[8] P. E. Kloeden and E. Platen. Numerical solution of stochastic differential equations, volume 23 of Applications of Mathematics. Springer, corrected third printing. edition, 1999.

[9] E. Levine and T. Hwa. Stochastic fluctuations in metabolic pathways. PNAS, 104:92249229, 2007.

[10] The MathWorks, Inc. - MATLAB and Simulink for Technical Computing: homepage. Retrieved June 5, 2007, from http: / / www . mathworks . com/, 2007.

[11] S. Paliwal, L. Ma, J. Krishnan, A. Levchenko, and P. A. Iglesias. Responding to directional cues: a tale of two cells. IEEE Control Systems Magazine, pages 77-90, August 2004.

[12] C. V. Rao, D. M. Wolf, and A. P. Arkin. Control, exploitation and tolerance of intracellular noise. Nature, 420(14):231-237, November 2002.

[13] R. Steuer. Effects of stochasticity in models of the cell cycle: from quantized cycle times to noise-induced oscillations. Journal of Theoretical Biology, 228:293-301, 7 June 2004.

[14] R. Steuer, C. Zhou, and J. Kurths. Constructive effects of fluctuations in genetic and biochemical regulatory systems. BioSystems, 72:241-251, 2003.

[15] D. Thorwarth, S. M. Eschmann, F. Paulsen, and M. Alber. A kinetic model for dynamic [18F]-Fmiso PET data to analyse tumour hypoxia. Physics in Medicine and Biology, 50:2209-2224, 2005.

[16] B. A. A. Weaver and D. W. Cleveland. Decoding the links between mitosis, cancer, and chemotherapy: The mitotic checkpoint, adaptation, and cell death. Cancer Cell, 8(1):7$12,2005$. 
[17] D. Zhang, M. Li, W. Ma, Y. Hou, Y.-H. Li, S.-W. Li, Q.-Y. Sun, and W.-H. Wang. Localization of mitotic arrest deficient 1 (MAD1) in mouse oocytes during the first meiosis and its functions as a spindle checkpoint protein. Biol Reprod, 72(1):58-68, 2005. 